American Journal of Biochemistry and Biotechnology 2 (2): 66-72, 2006

ISSN 1553-3468

(C) 2006 Science Publications

\title{
Gene Technology in Tissue Engineering
}

\author{
${ }^{1}$ Xiao-Dan Sun and ${ }^{2}$ In-Seop Lee \\ ${ }^{1}$ Laboratory of Advanced Materials, Department of Materials Science \& Engineering, \\ Tsinghua University, Beijing 100084, China \\ ${ }^{2}$ Institute of Physics \& Applied Physics, and Atomic-scale Surface Science Research Center, \\ Yonsei University, Seoul 120-749, Korea
}

\begin{abstract}
Scaffold, cells and signaling factors are regarded as the three essential components in tissue engineering. With the development of molecular and cell biology, gene technology is beginning to show a promising position in tissue engineering as it can influence these essential components at DNA-level. By introducing plasmid DNA or genes encoding certain signaling factors (growth factors/cytokines) into the cells, required growth factors/cytokines can be expressed and secreted spatially and temporally by the transfected cells, which will promote the differentiation, proliferation and organization of the cells on the scaffold. Protein-based scaffolds which have specific structures can also be prepared genetically to induce attachment and spreading of the cells. This paper reviews research work of gene technology developed in tissue engineering.
\end{abstract}

Key words: Gene engineering, tissue engineering, molecular biology, cell biology

\section{INTRODUCTION}

Tissue engineering refers to the science of generating new living tissues to replace, repair or augment the diseased/damaged tissue and restore tissue/organ function ${ }^{[1]}$. Regardless of the preparation technique, tissue engineering requires at least three key components, which are scaffold/matrix to support cells for the tissue formation, responsive cells to produce cellular and extracellular matrix and growth-inducing stimulus factors (signaling factors) to promote the division, maturation and differentiation of the cells ${ }^{[2-4]}$. Accordingly, the improvement of scaffold design, spatiotemporal delivery/release of signaling factors and subsequent cell proliferation remain the challenges of tissue engineering.

With the development of cell biology and molecular biology, gene technology represents a promising method to meet these challenges of tissue engineering applications in several different ways by controlling the synthesis of the scaffolds or the secretion of the signaling factors at DNA-level (Figure 1). New fields of 'gene-enhanced tissue engineering' (Route (1) and (2) in Figure 1) ${ }^{[5-8]}$ and 'genetically engineered protein-based polymers' (Route (3) in Figure 1) [9] have emerged as attractive subjects in tissue engineering. In this review, we will summarize some work in these developing fields to show the potentially promising prospects of the combination between tissue engineering and gene technology.

Corresponding Author: In-Seop Lee, Institute of Physics \& Applied Physics, and Atomic-scale Surface Science Research Center, Yonsei University, Seoul 120-749, Korea

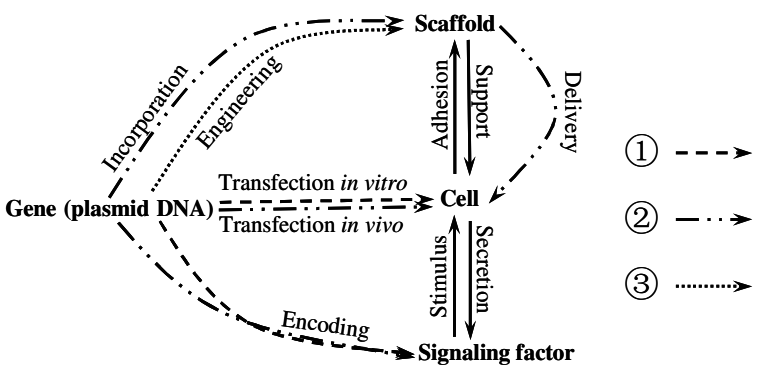

Fig. 1: Illustration of different ways in which gene technology is used in tissue engineering. Route (1): ex vivo approach for gene-enhanced tissue engineering; Route (2): in vivo approach for gene-enhanced tissue engineering; Route (3): genetically engineered scaffold materials.

\section{GENE-ENHANCED TISSUE ENGINEERING}

The most common approach to tissue engineering is to seed cells derived from either biopsies or stem cells on biodegradable scaffolds configured to the shape of the new tissue in vitro, and then implant the cellseeded scaffolds into the patient subsequently when the cells are differentiated and proliferated to certain extent ${ }^{[10]}$. Additionally, the presence of high and sustained levels of signaling factors has been demonstrated to be one of the most important factors for in vivo healing since the motility, differentiation, organization, proliferation and apoptosis of the cells associated with tissue formation are greatly influenced by soluble (e.g. cytokines) and insoluble (e,g. ECM proteins) factors in the local microenvironment ${ }^{[11,12]}$. The most obvious 
means of delivering these signaling factors (growth factors or cytokines) in situ is direct application of the recombinant protein ${ }^{[13]}$. However, most growth factors are rapidly cleared and have half-lives of minutes ${ }^{[12]}$, and dosage of milligram amounts of recombinant cytokine is required to show significant efficacy advantages, which is expensive to manufacture and presents an additional safety risk ${ }^{[5]}$. Thereby, the ability to regulate expression of desired signaling factors in time and space may be critical to the engineering of complex tissue architectures. On this occasion, 'geneenhanced tissue engineering' has turned up to be a promising way to provide sustained delivery of growth factor/cytokine to a local site in vivo. An important assumption herein was that, following gene transfer, the recombinant cytokine would be expressed at more nearly physiological levels but for a prolonged period of time by local transfected cells.

\subsection{Categories of Gene-Enhanced Tissue Engineering}

Gene-enhanced tissue engineering deals with the scientific and technologic endeavors to produce cultured cells or polymer matrices transduced with defined gene vectors encoding certain signaling factors by means of genetic engineering technique, to make transduced cells or gene activated matrices (GAM) highly express corresponding cytokines/growth factors into the microenvironment, and then to enhance the differentiation and proliferation abilities of the cells to finally construct artificial tissue with desired functions [14].

Gene-enhanced tissue engineering can be divided into two categories according to the approaches for the gene transfer, which are ex vivo approach and in vivo approach ${ }^{[2,14,15]}$. The ex vivo approach illustrated in Route (1) in Figure 1 consists of isolating cells from a tissue biopsy, transferring a defined gene/plasmid DNA encoding required signaling factors into cells using viral or non-viral vectors and growing cells in culture for them to be transfected or transduced in vitro before the cells are seeded into the matrix and the cellsupplemented matrix is finally implanted into the patient. The alternative in vivo approach illustrated in Route (2) in Figure 1 consists of directly injecting genevector or implanting the gene-supplemented matrix to relevant position to transduce the cells in situ by the delivery and release of the gene/plasmid DNA within the patient. In both approaches, the expression and secretion of desired signaling factors by the transduced cells are expected to stimulate and enhance the survival, proliferation or function of particular populations of cells in return.

Up to now, the ex vivo approach is presented to be safe and effective, while the techniques are complicated since it requires appropriate cell sourcing and potentially more intensive surgical procedures, such as harvesting of autologous cells or implantation of cellscaffold constructs ${ }^{[16]}$. On the other hand, the in vivo approach is attractive because of its technical simplicity such as the controlled releasing of DNA. However, the in vivo approach is presently limited by the demonstration of both safety and biological efficacy ${ }^{[15]}$. It has great potential to be used in the setting of inherited genetic defects and in tissues that consist of relatively homogeneous long-lived cells and in which phenotypic expression may be induced and durably maintained by expression of a single gene ${ }^{[17]}$.

\subsection{Applications of Gene-Enhanced Tissue Engineering}

Since 1993, Bonadio's research group has explored the possibility that cytokines and growth factors were delivered not as recombinant proteins but as plasmidgenes ${ }^{[18]}$. Till now, as a means of delivering growth factors or cytokines with cells in a scaffold to the sites of tissue injury to accelerate and/or induce a natural biological regeneration, gene technology has been developed in tissue engineering for many tissues and organs such as bone ${ }^{[4,15,19]}$, cartilage ${ }^{[20-22]}$, tendon, ligament [23, 24], muscle ${ }^{[2]}$, heart ${ }^{[25,26]}$, skin ${ }^{[27,28]}$, neuron ${ }^{[28]}$ and et al ${ }^{[29-31]}$. Herein, we will summarize some of the research work on the application of geneenhanced tissue engineering for these tissues according to the gene transfer approaches described above.

\subsubsection{Applications of ex vivo approach}

The ex vivo approach was the initial effort toward developing somatic gene therapy. A large number of cell types have been proposed to be transduced by this $\operatorname{approach}^{[32]}$.

As bone morphogenetic protein (BMP) is one of the most important cytokines that control the cellular events associated with bone formation and repair ${ }^{[33]}$, a variety of BMP-transduced cell types, including osteoblasts, bone marrow stromal, muscle-derived, periosteal, and fibroblastic cells, have shown to produce bone in ectopic sites ${ }^{[34-41]}$. In 1999, Breitbart and Mason et al. demonstrated that cultured rabbit periosteal cells transduced retrovirally with the bone morphogenetic protein 7 (BMP-7) gene could be produced, and polyglycolic acid (PGA) matrices seeded with these cells could repair critical-size rabbit cranial defects much better than the negative controltransduced cells/PGA, non-transduced cells/PGA or PGA alone ${ }^{[7]}$. They used the similar scheme to transduce and culture periosteal-derived rabbit mesenchymal stem cells with the BMP-7 gene, and the PGA grafts containing these modified cells consistently showed complete or near complete bone and articular 
cartilage regeneration at 8 and 12 weeks. This is the first report of articular cartilage regeneration using a combined gene therapy and tissue engineering approach ${ }^{[6]}$. Lou and collaborators showed that BMP-2engineered allogenic mesenchymal stem cells repair critically sized rat femoral defects to the same degree as engineered autologous cells, if the allogenic group received short-term immunosuppressant therapy ${ }^{[42]}$. The ex vivo approaches in bone and cartilage geneenhanced tissue engineering are not limited to BMP as expression of LIM mineralization protein-1 (LMP-1) and Runx2/Cbfa1 transcription factor promote bone formation in heterotopic and orthotopic sites ${ }^{[43-48]}$.

Exposure of de-endothelialized vascular structures to blood flow will cause platelet adherence and thrombus formation. Bare synthetic vascular graft material is highly thrombogenic because of serum protein adherence and platelet deposition. Thus, seeding of genetically modified endothelial cells onto vascular grafts has emerged as a promising technique within the cardiovascular system. In 1989, Zwiebel et al. reported high levels of recombinant gene expression in rabbit endothelial cells ${ }^{[49]}$. In the same year, Wilson et al. implanted synthetic vascular grafts seeded with retrovirally transduced endothelial cells in a canine model ${ }^{[50]}$. The t-PA transduced baboon endothelial cells seeded onto collagen coated vascular grafts reduced platelet and fibrin deposition in baboon femoral arteriovenous shunts ${ }^{[51]}$. However, it is also reported that the proteolytic effects of the t-PA secreted by the transduced endothelial cells might diminish the cells' ability to adhere to vascular graft material in vivo over time ${ }^{[52]}$, which reminds the researchers to pay more attention to the nature of the recombinant gene products being delivered by seeded endothelial cells ${ }^{[25]}$.

The ex vivo approach was also used in would healing models by Breitbart et al. The human plateletderived growth factor $b$ (PDGF-B) gene was introduced into primary rat dermal cells in vitro. Seeding of the gene-modified cells onto PGA scaffold matrices and introduction into the rat model resulted in substantially increased fibroblast hypercellularity over control wounds at both 7 and 14 days posttreatment. ${ }^{[8]}$.

\subsubsection{Applications of in vivo approach}

In order to introduce DNA directly into tissues, different methods have been tried to locally deliver plasmid gene constructs. For example, DNA has been formulated in a liquid buffer (e.g. " naked DNA') ${ }^{\text {[53-55] }}$ and with (proteo)liposome carriers ${ }^{[56,57]}$, has been encapsulated in controlled release synthetic polymer particles ${ }^{[58]}$, has been incorporated into hydrogels ${ }^{[59]}$ and sustained release polymer emulsions ${ }^{[60]}$. All the above methods belong to polymeric release, in which DNA is entrapped within the material and released into the environment, with release typically occurring through a combination of diffusion and polymer degradation $^{[61]}$.

As to the gene delivery in vivo in tissue engineering, substrate-mediated delivery was developed, in which DNA is concentrated at the delivery site and targeted to the cells that are adhered to the substrate ${ }^{[62,63]}$. Cells cultured on the substrate can internalize the DNA either directly from the surface or by degrading the linkage between the vector and the material. The structural matrix in which DNA has been incorporated directly is called as gene activated matrices (GAM) ${ }^{[5,11,32,61,64,65]}$ The matrices in GAM then act in a dual role of both structural support for cell growth and vehicle for controlled release of tissue inductive factors. The first GAM feasibility study involved direct plasmid-gene transfer to repair cells participating in bone repair in the adult rat ${ }^{[66]}$. GAMs with either a BMP-4 plasmid or a plasmid coding for a secreted fragment of parathyroid hormone (PTH) induced in a biological response of new bone filling the defect. Implantation of a two-plasmid GAM (BMP-4 plus PTH 1-34, which act synergistically) caused new bone to form faster than with either factor alone. This work showed for the first time that new bone will form rapidly in vivo following direct osteoinductive plasmid gene transfer to fibroblasts.

GAM system has been used in a variety of natural and synthetic materials for DNA delivery in different tissues. Collagen-based delivery of nonviral or viral DNA has been employed in models of bone ${ }^{[66-68]}$, cartilage ${ }^{[69]}$, and nerve regeneration ${ }^{[70]}$, wound healing ${ }^{[71-73]}$, muscle repair ${ }^{[74]}$ and cardiovascular disease ${ }^{[75]}$. Porous poly(lactide-co-glycolide) (PLG) scaffolds releasing plasmid DNA were able to transfect cells within and around the scaffold, with sufficient expression of PDGF to promote tissue formation ${ }^{[76]}$.

In summary, gene-enhanced tissue engineering studies have illustrated the potential for extending the production of growth factors locally. Adapting the gene strategies to control the expression of the signaling factors spatially (micrometers to millimeters) or temporally (days to months) may recreate the environmental complexity present during tissue formation, which will promote the efficacy of tissue engineering.

\section{GENETICALLY ENGINEERING SCAFFOLD MATERIALS}

As mentioned above, the scaffold used in tissue engineering acts to support cell colonization, migration, growth and differentiation, and often guides the development of the required tissue. Rutherford et al. showed that different scaffolds support in vivo bone formation to various degrees ${ }^{[77]}$. This study suggests that the nature and properties of the scaffold plays an 
important role in bone engineering, and the scaffold can be engineered to help to optimize cell delivery and tissue formation.

The nature and biological fate of the scaffolds, which are usually natural or synthetic polymers, depends on their molecular architecture. The molecular weight, composition, sequence and stereochemistry of chemically synthesized polymers are usually heterogeneous and defined in terms of statistical distributions ${ }^{[78]}$. With the progress of gene technology, genetic engineering methodology has enabled the synthesis of protein-based polymers with precisely controlled structures ${ }^{[78-83]}$. In contrast to chemically synthesized poly(amino acid)s and sequential polypeptides, the entire amino acid sequence of genetically engineered polymers is controlled at the DNA-level, leading to polymers with precisely defined, and potentially quite complex, sequences and structures. Protein-based polymers can be designed to incorporate a variety of functionalities, including responsiveness to microenvironmental stimuli, controlled biodegradation and the presentation of informational motifs for cellular and subcellular interactions ${ }^{[9]}$. Varieties of polymers, such as Elastinlike polymers, silk-like polymers, silk-elastinlike block copolymers, coiled-coil and leucin-rich protein domains, $\square$-sheet forming polymers, alanylglycine polymers and recombinant poly(glutamic acid) polymers, have been produced using the genetic engineering method ${ }^{[9]}$. The genetically engineered protein-based polymers have been applied in blood vessel reconstruction to improve the attachment and spreading ability of the endothelial cells ${ }^{[84]}$. Artificial extracellular matrix protein composed of a repeating unit structure [GVPGI])x and a cell-binding domain (designated CS5) derived from the natural extracellular matrix protein fibronectin were suggested as a vascular graft material. It was demonstrated that spreading of cells was enhanced on surfaces coated with $\left[\mathrm{CS}_{5}(\mathrm{GVPGI})_{20}\right]_{5}$, when compared to surfaces coated with fibronectin.

Although no many reports can be found yet in the field of genetically engineered scaffold materials at present time, the authors suppose that it may become another attractive topic in advanced tissue engineering.

\section{FINAL REMARKS}

As a multidisciplinary research area that incorporates both biological and engineering principles, tissue engineering has the potential to develop widely with the fast evolvement in biology science. The confluence of molecular and cell biology, materials science and engineering provides the tools to create controllable microenvironments that mimic natural developmental processes and direct tissue formation for experimental and therapeutic applications. The results of recent efficacy studies provide evidence that delivery of certain stimulatory gene products can be used to elicit favorable biological responses in vivo. However, the development of gene technology in tissue engineering will have to experience more experiments and validation before it becomes a practical therapy method as there still exist many challenges and/or unclear understandings in this field of science.

E. Pennisi ${ }^{[85]}$ once pointed out that although genes have played biology's center stage for decades as the units of DNA that define the proteins needed for life, gene expression is not determined solely by the DNA code itself. Gene activity also depends on a host of socalled epigenetic phenomena and can be altered by proteins and RNA. Deeper understanding of the role of DNA with the evolvement of biology science will definitely improve the using of gene technology in tissue engineering radically.

The two major challenges facing gene technology in tissue engineering presently include the problem of identifying appropriate genes that are effective in tissue repair based on delineation of the protein needed for proper tissue function, and the reliable expression of the therapeutic gene for achieving effective repair of diseased or injured tissue in a durable manner ${ }^{[20,86]}$. More sophisticated promoter systems may be incorporated to finely tune the transgenic expression process if the required quantity and time of expression can be determined. A sustained track record of safety and long-term clinical efficacy should be established prior to human testing, since many of the gene products implicated for use in treatment have the potential for generating significant side effects ${ }^{[20]}$.

Considering the engineering of complex tissue architectures, such as those found in vascular networks and the nervous systems, the ability to regulate expression of one or more factors in time and space may be critical. Studies in these systems would also increase our understanding of the biology behind tissue formation, which would serve to identify how gene delivery can best augment the regenerative process ${ }^{[61]}$.

In summary, gene technology has shown a promising potential to be used in tissue engineering for the optimal tissue regeneration, yet, much work has to be carried out to consummate the knowledge and techniques in this field.

\section{ACKNOWLEDGMENTS}

We acknowledge the financial support from both the project No. 50402001 supported by National Natural Science Foundation of China, and the grant (code \#: 06K1501-01620) from Center for Nanostructured Materials Technology under 21st Century Frontier R\&D Program of the Ministry of Science and Technology, Korea. 


\section{REFERENCES}

1. A. Curtis, M. Riehle, 2001. Tissue engineering: the biophysical background. Physics in Medicine and Biology, 46 (4): R47-R65.

2. Vonda J. Wright, Hairong Peng and Johnny Huard, 2001. Muscle-based gene therapy and tissue engineering for the musculoskeletal system, DDT, 6 (14): 728-733.

3. G. F. Muschler, R. J. Midura, 2002. Connective tissue progenitors: practical concepts for clinical applications. Clin. Orthop. 395: 66-80.

4. M. Nakashima, A. Akamine, 2005. The application of tissue engineering to regeneration of pulp and dentin in endodontics. Journal of Endodontics 31 (10): 711-718.

5. J. Bonadio, 2000. Tissue Engineering via Local Gene Delivery: Update and Future Prospects for Enhancing the Technology. Advanced Drug Delivery Reviews, 44: 185-194.

6. J. M. Mason, A. S. Breitbart, M. Barcia, D. Porti, R. G. Pergolizzi, D. A. Grande, 2000. Cartilage and Bone Regeneration Using Gene-Enhanced Tissue Engineering. Clinical Orthopaedics and Related Research, 379: 171-178.

7. A. S. Breitbart, D. A. Grande, J. M. Mason, M. Barcia, T. James, R. T. Grant, 1999. GeneEnhanced Tissue Engineering: Applications for Bone Healing Using Cultured Periosteal Cells Transduced Retrovirally with the BMP-7 Gene. Annals of Plastic Surgery, 42 (5): 488-495.

8. A. S. Breitbart, J. M. Mason, C. Urmacher, M. Barcia, R. T. Grant, R. G. Pergolizzi, D. A. Grande, 1999. Gene-Enhanced Tissue Engineering: Applications for Wound Healing Using Cultured Dermal Fibroblasts Transduced Retrovirally With the PDGF-B Gene. Annals of Plastic Surgery, 43 (6): 632-639.

9. M. Haider, Z. Megeed, H. Ghandehari, 2004. Genetically engineered polymers: status and prospects for controlled release. Journal of Controlled Release 95: 1-26.

10. R. Langer, J. P. Vacanti, 1993. Tissue engineering. Science, 260: 920-925.

11. D. M. Salvay, L. D. Shea, 2006. Inductive tissue engineering with protein and DNA-releasing scaffolds. Molecular Biosystems, 2 (1): 36-48.

12. Samuel P. Baldwin, W. Mark Saltzman, 1998. Materials for protein delivery in tissue engineering. Advanced Drug Delivery Reviews 33: 71-86.

13. R. Langer, 1998. Drug delivery and targeting. Nature, 392 (supp): 5-10.

14. J. Wu, H. Q. Chen, 2003. Research Advances in Gene-enhanced Tissue Engineering. Journal of Biomedical Engineering, 20 (4): 733-737.

15. Dietmar W. Hutmacher, Andres J. Garcia, 2005. Scaffold-based bone engineering by using genetically modified cells, Gene 347: 1-10.

16. Y. Gafni, G. Turgeman, M. Liebergal, G. Pelled, Z. Gazit, D. Gazit, 2004. Stem cells as vehicles for orthopedic gene theraphy. Gene Ther. 11: 417426.
17. G. F. Muschler, C. Nakamoto, L.G. Griffith, 2004. Engineering principles of clinical cell-based tissue engineering, J. Bone Jt. Surg., Am. 86-A (7): $1541-1558$.

18. J. Bonadio, 2000. Local gene delivery for tissue regeneration. E-Biomed 1: 25-29.

19. R. T. Franceschi, 2005. Biological approaches to bone regeneration by gene therapy, Journal of Dental Research 84 (12): 1093-1103.

20. S. B. Trippel, S. C. Ghivizzani and A. J. Nixon, 2004. Gene-based approaches for the repair of articular cartilage. Gene Therapy, 11, 351-359.

21. Ai-Guo Zhang, Qi-Rong Dong, 2005. Research Advances in geng-enhanced cartilage tissue engineering. Chinese Journal of Orthopaedic Trauma, 7 (5): 476-479.

22. V. Martinek, F. H. Fu, C. W. Lee, J. Huard, 2001. Treatment of osteochondral injuries - Genetic engineering. Clinics in Sports Medicine, 20 (2): 403-+.

23. D. Wu, P. Razzano and Daniel A. Grande, 2003. Gene Therapy and Tissue Engineering in Repair of the Musculoskeletal System. Journal of Cellular Biochemistry, 88: 467-481.

24. J. Huard, Y. Li, H. R. Peng, F. H. Fu, 2003. Gene therapy and tissue engineering for sports medicine. Journal of Gene Medicine, 5 (2): 93108.

25. Sahil A. Parikh, Elazer R. Edelman, 2000. Endothelial Cell Delivery for Cardiovascular Therapy. Advanced Drug Delivery Reviews, 42: 139-161.

26. Prisca Zammaretti and Marisa Jaconi, 2004. Cardiac tissue engineering: regeneration of the wounded heart. Current Opinion in Biotechnology. 2004, 15: 430-434.

27. S. T. Andreadis, 2004. Gene transfer to epidermal stem cells: implications for tissue engineering. Expert Opinion on Biological Therapy 4 (6): 783-800.

28. C. Thanos, D. Emerich, Delivery of neurotrophic factors and therapeutic proteins for retinal diseases. Expert Opinion on Biological Therapy, 5 (11): 1443-1452.

29. S. M. Warren, K. D. Fong, C. M. Chen, E. G. Loboa, C. M. Cowan, H. P. Lorenz, M. T. Longaker, 2003. Tools and techniques for craniofacial tissue engineering. Tissue Engineering, 9 (2): 187-200.

30. A. Vats, N. S. Tolley, J. M. Polak, B. C. Knight, 2002. Gene Expression: A Review of Clinical Applications in Otorhinolaryngology-Head and Neck Surgery. Clinical Otolaryngology, 27 (5): 291-295.

31. Dirk Schultheiss, 2004. Regenerative Medicine in Andrology: Tissue Engineering and Gene Therapy as Potential Treatment Options for Penile Deformations and Erectile Dysfunction. European Urology 46: 162-169.

32. Jeffrey Bonadio, Steven A. Goldstein, Robert J. Levy, 1998. Gene therapy for tissue repair and regeneration. Advanced Drug Delivery Reviews, 33: 53-69. 
33. A. Erlebacher, E. H. Filvaroff, S. E. Gitelman, R. Derynck, 1995. Toward a molecular understanding of skeletal development. Cell 80: 371-378.

34. P. H. Krebsbach, K. Gu, R. T. Franceschi, R. B. Rutherford, 2000. Gene therapy-directed osteogenesis: BMP-7-transduced human fibroblasts form bone in vivo. Hum. Gene Ther, 11: $1201-1210$.

35. C. T. Laurencin, et al., 2001. Poly(lactide-coglycolide)/hydroxyapatite delivery of BMP-2producing cells: a regional gene therapy approach to bone regeneration. Biomaterials, 22: 12711277.

36. J. R. Lieberman, et al., 1998. Regional gene therapy with a BMP-2-producing murine stromal cell line induces heterotopic and orthotopic bone formation in rodents. J. Orthop. Res. 16: 330339.

37. J. Lou, F. Xu, K. Merkel, P. Manske, 1999. Gene therapy: adenovirus-mediated human bone morphogenetic protein-2 gene transfer induces mesenchymal progenitor cell proliferation and differentiation in vitro and bone formation in vivo. J. Orthop. Res. 17: 43- 50.

38. J. M. Mason, D. A. Grande, M. Barcia, R. Grant, R. G. Pergolizzi, A. S. Breitbart, , 1998. Expression of human bone morphogenic protein 7 in primary rabbit periosteal cells: potential utility in gene therapy for osteochondral repair. Gene Ther. 5: 1098- 1104.

39. D. S. Musgrave, R. Pruchnic, P. Bosch, B. H. Ziran, J. Whalen, J. Huard, 2002. Human skeletal muscle cells in ex vivo gene therapy to deliver bone morphogenetic protein-2. J. Bone Jt. Surg., Br. 84: 120- 127.

40. K. Partridge, et al., 2002. Adenoviral BMP-2 gene transfer in mesenchymal stem cells: in vitro and in vivo bone formation on biodegradable polymer scaffolds. Biochem. Biophys. Res. Commun. 292: $144-152$.

41. G. Turgeman, et al., 2001. Engineered human mesenchymal stem cells: a novel platform for skeletal cell mediated gene therapy. J. Gene Med. 3: $240-251$.

42. H. Tsuchida, J. Hashimoto, E. Crawford, P. Manske, J. Lou, 2003. Engineered Allogeneic Mesenchymal Stem Cells Repair Femoral Segmental Defect in Rats. J. Orthop. Res. 21: 44- 53.

43. S. D. Boden, et al., 1998. Lumbar spine fusion by local gene therapy with a cDNA encoding a novel osteoinductive protein (LMP-1). Spine, 23: 2486-2492.

44. B. A. Byers, R. E. Guldberg, A. J. Garcia, 2004. Synergy between genetic and tissue engineering: Runx2 overexpression and in vitro construct development enhance in vivo mineralization. Tissue Engineering, 10: 1757- 1766.

45. M. Viggeswarapu, et al., 2001. Adenoviral delivery of LIM mineralization protein-1 induces new-bone formation in vitro and in vivo. J. Bone Jt. Surg., Am. Vol. 83-A: 364-376.
46. S. Yang, D. Wei, D. Wang, M. Phimphilai, P. H. Krebsbach, R. T. Franceschi, 2003. In vitro and in vivo synergistic interactions between the Runx2/Cbfa1 transcription factor and bone morphogenetic protein-2 in stimulating osteoblast differentiation. J. Bone Miner. Res. 18: 705- 715.

47. K. Hirata, et al., 2003. Transplantation of skin fibroblasts expressing BMP-2 promotes bone repair more effectively than those expressing Runx2. Bone, 32: 502-512.

48. H. Zheng, et al., 2004. Cbfa1/osf2 transduced bone marrow stromal cells facilitate bone formation in vitro and in vivo. Calcif. Tissue Int. 74: 194- 203.

49. J. A. Zwiebel, S. M. Freeman, P. W. Kantoff, K. Cornetta, U. S. Ryan, W. F. Anderson, 1989. High-level recombinant gene expression in rabbit endothelial cells transduced by retroviral vectors. Science, 243 (4888): 220-222.

50. J. M. Wilson, L. K. Birinyi, R. N. Salomon, P. Libby, A. D. Callow, R. C. Mulligan, 1989. Implantation of vascular grafts lined with genetically modified endothelial cells, Science 244 (4910): 1344-1346.

51. D. A. Dichek, J. Anderson, A. B. Kelly, S. R. Hanson, L. A. Harker, 1996. Enhanced in vivo antithrombotic effects of endothelial cells expressing recombinant plasminogen activators transduced with retroviral vectors, Circulation 93 (2): 301-309.

52. P .F. Dunn, K. D. Newman, M. Jones, I. Yamada, V. Shayani, R. Virmani, D. A. Dichek, 1996. Seeding of vascular grafts with genetically modified endothelial cells. Secretion of recombinant TPA results in decreased seeded cell retention in vitro and in vivo [see comments]. Circulation, 93 (7): 1439-1446.

53. J. A. Wolff, R. M. Malone, P. Williams, et al., 1990. Direct gene transfer into mouse muscle in vivo. Science, 247: 1465-1468.

54. J. A. Wolff, 1997. Naked DNA transport and expression in mammalian cells. Neuromuscul. Disord. 7: 314-318.

55. J. A. Norman, P. Hobart, M. Manthorpe, P. Felgner, C. Wheeler, 1997. Development of improved vectors for DNA-based immunization and other gene therapy applications. Vaccine, 15: 801-803.

56. P. L. Felgner, 1997. Nonviral strategies for gene therapy. Sci. Am. 276: 102-106.

57. R. I. Mahato, A. Rolland, E. Tomlinson, 1997. Cationic lipid-based gene delivery systems: pharmaceutical perspectives. Pharm. Res. 14: 853-859.

58. E. Mathiowitz, J. nS. Jacob, Y. S. Jong, et al., 1997. Biologically erodable microspheres as potential oral drug delivery systems. Nature, 386: 410-414.

59. J. M. Isner, J. F. Symes, K. Walsh, et al., 1996. Clinical evidence of angiogenesis after arterial gene transfer of phVEGF in patients with ischaemic limb. Lancet, 348: 370-374. 
60. J. Bonadio, E. Smiley, S. A. Goldstein, K. Ciftci, V. Labhasetwar, R. J. Levy, Direct gene transfer in vivo using the GAM technology. Cold Spring Harbor Gene Therapy Meeting, Cold Spring Harbor, NY, September 25-29, 1996.

61. Angela K. Pannier and Lonnie D. Shea, 2004. Controlled Release Systems for DNA Delivery. Molecular Therapy, 10 (1): 19-26.

62. R. J. Levy, et al. 2001. Localized adenovirus gene delivery using antiviral $\operatorname{IgG}$ complexation. Gene Ther. 8: 659-667.

63. T. Segura, and L. D. Shea, 2002. Surface-tethered DNA complexes for enhanced gene delivery. Bioconjugate Chem. 13: 621-629.

64. J. Bonadio, 2000. Tissue Engineering via Local Gene Delivery. Journal of Molecular MedicineJMM 78 (6): 303-311.

65. T. P. Richardson, W. L. Murphy, D. J. Mooney, 2001. Polymeric delivery of proteins and plasmid DNA for tissue engineering and gene therapy. Critical Reviews in Eukaryotic Gene Expression 11 (1-3): 47-58.

66. J. Fang, Y.-Y. Zhu, E. Smiley, J. Bonadio, J.A. Rouleau, S.A. Goldstein, L.K. McCauley, B. Davidson, B. Roessler, Stimulation of new bone formation by direct transfer of osteoinductive plasmid genes, Proc. Natl. Acad. Sci. USA 93 (1996) 5753-5758.

67. J. Bonadio, E. Smiley, P. Patil, and S. Goldstein, 1999. Localized, direct plasmid gene delivery in vivo: prolonged therapy results in reproducible tissue regeneration. Nat. Med. 5: 753 - 759.

68. V. Labhasetwar, J. Bonadio, S. A. Goldstein, and J. R. Levy, 1999. Gene transfection using biodegradable nanospheres: results in tissue culture and a rat osteotomy model. Colloids Surf. B 16: 281- 290.

69. A. Pascher, et al. 2004. Gene delivery to cartilage defects using coagulated bone marrow aspirate. Gene Ther. 11: 133 - 141 .

70. M. Berry, et al. 2001. Sustained effects of geneactivated matrices after CNS injury. Mol. Cell. Neurosci. 17: 706 - 716.

71. J. Doukas, et al. 2001. Matrix immobilization enhances the tissue repair activity of growth factor gene therapy vectors. Hum. Gene Ther. 12: 783 798.

72. L. A. Chandler, et al. 2000. FGF2-targeted adenovirus encoding platelet-derived growth factor-B enhances de novo tissue formation. Mol. Ther. 2: $153-160$.

73. D. Gu, T. Nguyen, M. L. Phillips, L. A. Chandler, and B. A. Sosnowski, 2004. Matrix-immobilized growth factor gene therapy enhances tissue repair. Wounds, 16: 34-41.

74. J. Doukas, et al. 2002. Delivery of FGF genes to wound repair cells enhances arteriogenesis and myogenesis in skeletal muscle. Mol. Ther. 5: 517527.
75. B. D. Klugherz, et al. 2000. Gene delivery from a DNA controlled-release stent in porcine coronary arteries. Nat. Biotechnol. 18: 1181-1184.

76. L. D. Shea, E. Smiley, J. Bonadio, and D. J. Mooney, 1999. DNA delivery from polymer matrices for tissue engineering. Nat. Biotechnol. 17: 551-554.

77. R. B. Rutherford, M. Moalli, R. T. Franceschi, D. Wang, K. Gu, P. H. Krebsbach, 2002. Bone morphogenetic protein-transduced human fibroblasts convert to osteoblasts and form bone in vivo. Tissue Eng. 8: 441-452.

78. K. P. McGrath, D. A. Tirrell, M. Kawai, T. L. Mason, M. J. Fournier, 1990. Chemical and biosynthetic approaches to the production of novel polypeptide materials, Biotechnol. Prog. 6 (3): 188-192.

79. J. Cappello, J. Crissman, M. Dorman, M. Mikolajczak, G. Textor, M. Marquet, F. Ferrari, 1990. Genetic engineering of structural protein polymers. Biotechnol. Prog. 6 (3): 198-202.

80. M. J. Fournier, H. S. Creel, M. T. Krejchi, T. L. Mason, D. A. Tirrell, K. P. McGrath, E. D. T. Atkins, 1991. Genetic synthesis of periodic protein materials. J. Bioact. Compat. Polym. 6 : 326-338.

81. A. E. Barron, R. N. Zuckermann, 1999. Bioinspired polymeric materials: in-between proteins and plastics. Curr. Opin. Chem. Biol. 3 (6): $681-687$.

82. D. E. Meyer, A. Chilkoti, 2002. Genetically encoded synthesis of protein-based polymers with precisely specified molecular weight and sequence by recursive directional ligation: examples from the elastin-like polypeptide system. Biomacromolecules, 3 (2): 357- 367.

83. R. A. McMillan, T. A. T. Lee, V. P. Conticello, 1999. Rapid assembly of synthetic genes encoding protein polymers. Macromolecules, 32 (11): 3643-3648.

84. A. Panitch, T. Yamaoka, M. J. Fournier, T. L. Mason, D. A. Tirrell, 1999. Design and biosynthesis of elastin-like artificial extracellular matrix proteins containing periodically spaced fibronectin S5 domains. Macromolecules, 32 (5): 1701-1703.

85. E. Pennisi, 2001. Behind the Scenes of Gene Expression. Science, 293: 1064-1067.

86. C. Andree, M. Kullmer, A. Wenger, D. J. Schaefer, U. Kneser, G. B. Stark, 2002. Gene technology and tissue engineering. Minimally Invasive Therapy \& Allied Technologies, 11 (3): 93-99. 